\title{
Estruturação de um guia interativo sobre as recomendações para um atendimento odontológico seguro e precoce aos pacientes com demência
}

\author{
Structuring an interactive guide on recommendations for safe and early dental care for patients \\ with dementia \\ Estructuración de una guía interactiva sobre recomendaciones para una atención odontológica \\ segura y temprana para pacientes con demencia
}

\author{
Érica Paula Barbosa \\ ORCID: https://orcid.org/0000-0002-8280-4699 \\ Universidade Estadual de Ciências da Saúde de Alagoas, Brasil \\ E-mail: erica.barbosa@ academico.uncisal.edu.br \\ Paulla Valéria de Souza Meneses \\ ORCID: https://orcid.org/0000-0001-6749-9377 \\ Universidade Estadual de Ciências da Saúde de Alagoas, Brasil \\ E-mail: paullavaleria@ hotmail.com \\ Cinthya Rafaella Magalhães da Nóbrega Novaes \\ ORCID: https://orcid.org/0000-0003-4795-6328 \\ Universidade Estadual de Ciências da Saúde de Alagoas, Brasil \\ E-mail: rafamn20@yahoo.com.br \\ Ana Renata Lima Leandro \\ ORCID: https://orcid.org/0000-0003-2707-0730 \\ Universidade Estadual de Ciências da Saúde de Alagoas, Brasil \\ E-mail: releandro@gmail.com \\ Monique Carla da Silva Reis \\ ORCID: https://orcid.org/0000-0002-8815-3938 \\ Universidade Estadual de Ciências da Saúde de Alagoas, Brasil \\ E-mail: moniquecsto@gmail.com
}

\begin{abstract}
Resumo
Considerando as necessidades de ampliar o alcance de educação em saúde para a população afetada pelas demências, foi elaborado um produto educacional de fácil divulgação, um guia tutorial interativo, com o título de "Práticas de saúde bucal em idosos com demência" que procurou incorporar a tecnologia para facilitar o processo ensino aprendizagem. Foi estruturado baseado no método CTM3 onde são didaticamente agrupados em etapas e baseados em três teorias, Concepção do produto, Referencial Teórico e Referencial Metodológico. O referencial teórico abrange o alicerce teórico sobre o assunto e tipo de produto a ser desenvolvido, demonstra sua relevância e utilidade e fundamenta sua concepção. A elaboração do produto educacional seguiu as seguintes etapas: concepção da ideia, escolha do público-alvo, estabelecimento do objetivo, e estabelecimento do orçamento. Em seguida foram definidos as etapas e os temas a serem abordados, estrutura de textos, bem como definição dos vídeos a serem incluídos e de artigos para fundamentar o tema exposto. O conteúdo explorado foi baseado na literatura científica relacionado aos cuidados e práticas de saúde bucal utilizada em idosos demenciados. O produto foi validado por banca de defesa de mestrado, sem sugestões de alteração do seu formato original, onde foi emitido o parecer validado sem alterações, a versão final foi publicada no Portal EduCAPES. Esta proposta de produto educacional apresenta instrumentos que buscam facilitar o processo de ensino-aprendizagem, através do estabelecimento de práticas educativas, instruindo cuidadores e dentistas quanto as práticas de saúde bucal em pacientes com demência utilizando a tecnologia como moderador do processo.
\end{abstract}

Palavras-chave: Produtos educacionais; Educação em saúde; Demência; Guia tutorial interativo.

\begin{abstract}
Considering the need to expand the reach of health education for the population affected by dementia, an educational product of easy dissemination was created, an interactive tutorial guide, entitled "Oral health practices in elderly people with dementia" that sought to incorporate the technology to facilitate the teaching-learning process. It was structured based on the CTM3 method where they are didactically grouped into stages and based on three theories, Product Design, Theoretical Framework and Methodological Framework. The theoretical framework covers the theoretical foundation on the subject and type of product to be developed, demonstrates its relevance and usefulness and underpins its design. The elaboration of the educational product followed the following steps: conception of the
\end{abstract}


idea, choice of the target audience, establishment of the objective, and establishment of the budget. Then, the steps and topics to be addressed, text structure, as well as definition of videos to be included and articles to support the exposed theme were defined. The content explored was based on the scientific literature related to oral health care and practices used in elderly people with dementia. The product was validated by a master's defense committee, with no suggestions to change its original format, where the validated opinion was issued without changes, the final version was published on the EduCAPES Portal. This educational product proposal presents instruments that seek to facilitate the teaching-learning process, through the establishment of educational practices, instructing caregivers and dentists about oral health practices in patients with dementia using technology as a moderator of the process.

Keywords: Educational products; Health education; Insanity; Interactive tutorial guide.

\section{Resumen}

Considerando la necesidad de ampliar el alcance de la educación en salud para la población afectada por demencia, se creó un producto educativo de fácil difusión, una guía tutorial interactiva, denominada "Prácticas de salud bucal en adultos mayores con demencia" que buscaba incorporar la tecnología para facilitar el proceso de enseñanzaaprendizaje. Se estructuró en base al método CTM3 donde didácticamente se agrupan en etapas y en base a tres teorías, Diseño de Producto, Marco Teórico y Marco Metodológico. El marco teórico cubre la fundamentación teórica sobre el tema y tipo de producto a desarrollar, demuestra su pertinencia y utilidad y sustenta su diseño. La elaboración del producto educativo siguió los siguientes pasos: concepción de la idea, elección del público objetivo, establecimiento del objetivo y establecimiento del presupuesto. Luego, se definieron los pasos y temas a abordar, la estructura del texto, así como la definición de videos a incluir y artículos de apoyo a la temática expuesta. El contenido explorado se basó en la literatura científica relacionada con el cuidado de la salud bucal y las prácticas utilizadas en personas mayores con demencia. El producto fue validado por un comité de defensa de maestría, sin sugerencias para cambiar su formato original, donde se emitió el dictamen validado sin cambios, la versión final fue publicada en el Portal EduCAPES. Esta propuesta de producto educativo presenta instrumentos que buscan facilitar el proceso de enseñanza-aprendizaje, a través del establecimiento de prácticas educativas, instruyendo a cuidadores y odontólogos sobre prácticas de salud bucal en pacientes con demencia utilizando la tecnología como moderador del proceso.

Palabras clave: Productos educativos; Educación para la salud; Locura; Guía tutorial interactiva.

\section{Introdução}

A senescência é o processo natural do envelhecimento, o qual compromete gradualmente os aspectos físicos, cognitivos e emocionais, acumulando um conjunto de fatores genéticos programados, ou seja, ocorre uma degradação funcional generalizada e progressiva, como consequência de perdas quanto a respostas de diferentes estímulos às circunstâncias estressoras, ampliando o número de risco de doenças associadas à velhice (Cancela, 2007).

$\mathrm{O}$ aumento da expectativa de vida, a redução das taxas de natalidade e a ampliação da assistência à saúde foram responsáveis pelo crescimento da população idosa brasileira (Paim et al., 2011), comumente acometida por doenças crônicas não transmissíveis (DCNT) que podem afetar a sua funcionalidade, repercutindo no desempenho das atividades cotidianas de forma independente, comprometendo de forma significativa a qualidade de vida dos idosos (Brasil, 2006).

As chances de a demência ocorrer aumentam com o avanço da idade. A probabilidade de ocorrência chega em $10 \%$ a mais em cada ano de vida, sendo sua prevalência maior entre as mulheres. No mundo, as demências acometem por volta de 50 milhões de indivíduos acima dos 65 anos, sendo registrados cerca 10 milhões de casos novos ao ano. Segundo cálculos, esta patologia poderá atingir 82 milhões de pessoas em 2030, chegando a 152 milhões em 2050 (OMS, 2019).

O envelhecimento é o maior fator de risco para a demência e com o avançar da idade aumenta a taxa de prevalência (OMS, 2019). O comprometimento cognitivo tem natureza progressiva e se torna ainda mais evoluído em estágios avançados, quando ocorrem alterações psicocomportamentais, disfunção motora e restrições físicas. Essas combinações resultam em várias deficiências nos cuidados de saúde e no autocuidado dos idosos com demência, incluindo higiene bucal e atendimento odontológico (Grossberg, 2003; Zenthofer et al., 2014; Fereshtehnejad et al. 2018).

O diagnóstico das demências, principalmente a Demência de Alzheimer (DA), na prática clínica, define-se, no máximo, como possibilidade ou probabilidade. De acordo com estudos atuais, somente o anatomopatológico seria definitivo (Laginestra-Silva et al. 2021). 
As circunstâncias da saúde oral podem contribuir no diagnóstico da demência senil, à proporção que constata a dificuldade da higienização bucal, já que um dos indicativos dessa enfermidade é a ausência do autocuidado (Brunetti \& Montenegro, 2002). Indivíduos com comprometimento cognitivo apresentam piores condições de saúde bucal quando comparados aos idosos com condição cognitiva preservada. Neste cenário, a privação de memória e a redução da motricidade provocada pelo adoecimento levam a redução dos cuidados relacionados à cavidade oral resultando em pobre situações de saúde bucal devido a associação entre higiene bucal deficiente e a incapacidade de reportar satisfatoriamente alterações bucais (Newton, 2007).

O tratamento odontológico deve ser iniciado o mais breve possível, assim que o diagnóstico seja firmado, pois com o progresso da doença, há crescente oposição da aceitação e realização do atendimento odontológico (Santiago, Simões, \& Pereira, 2008). Diversos aspectos devem ser levados em consideração durante o manejo clínico-odontológico de idosos com DA, uma vez que a doença apresenta desafios ao seu cuidado clínico e manejo (Miranda et al., 2018), e que a abordagem tem direta relação com o estado de avanço da doença.

O processo de educação em saúde deve ser entendido como um componente e um recurso a ser utilizado como estratégia no âmbito da promoção da saúde para melhor qualidade de vida do usuário (Brasil, 2007), independente da condição que ele apresente.

Franco et al. (2007) mostraram em seu estudo experiências no dia a dia de uso de mídias interativas no processo de edificação de conhecimento dos indivíduos e de elaboração de conteúdo digital, onde tais experiências demonstraram ser congruentes, práticas e justificável na formação técnica de indivíduos. Em situações em que a interatividade é importante, como apresentação de documentos, áudio, vídeo, exercícios em grupo, o manual interativo tem destaque (Boulet, 2011).

As tecnologias de informação abrangem todos os serviços desenvolvidos na sociedade pelos recursos da informática. Trata-se da disseminação social de conhecimentos em larga escala de propagação, a partir destes sistemas tecnológicos inteligentes. A informática é uma expressão simbólica em todas as suas variantes, transmitindo conhecimentos à sociedade (Souza et al., 2011).

A partir dos resultados dos estudos analisados, na revisão sistemática de Barbosa et al. (2021), considerando as necessidades de ampliar o alcance de educação em saúde para a população afetada pelas demências, foi elaborado um produto educacional de fácil divulgação, um guia tutorial interativo, com o título de "Práticas de saúde bucal em idosos com demência" que procurou incorporar a tecnologia para facilitar o processo ensino aprendizagem.

\section{Metodologia}

O percurso metodológico para a criação do Guia Interativo, produto educacional, vai ser descrito nos tópicos subsequentes. São eles: estruturação do guia tutorial, referencial teórico, referencial metodológico e validação do produto.

\subsection{Estruturação do Guia Interativo}

O Guia Interativo foi estruturado no método CTM3, onde de acordo com Santos e Warren (2020) esse método é facilitador na estruturação desse produto, possibilitando a maior inserção de elementos que farão parte da composição dele. Os elementos que compõem o Método CTM3 são didaticamente agrupados em três etapas e baseados em três teorias, Concepção do produto, Referencial Teórico e Referencial Metodológico (referenciado em três teorias).

\subsection{Referencial Teórico}

O referencial teórico abrange o alicerce teórico sobre o assunto e tipo de produto a ser desenvolvido, demonstra sua relevância e utilidade e fundamenta sua concepção. 


\subsection{Referencial metodológico utilizado}

\subsubsection{O Que é um Guia Interativo?}

Procurando integrar a tecnologia ao contexto do trabalho em saúde foi criado um guia tutorial interativo sobre Práticas de Saúde Bucal em idosos com demência onde através da tecnologia educacional, cria-se um acesso rápido e simples às informações, sendo uma nova maneira de ampliar o conhecimento, com novas ideias e valores. O educador em saúde tem que se preocupar que as informações adquiridas através dessa tecnologia educacional sejam úteis para que o público amplie essa aprendizagem sobre as informações transmitidas, ajudando a encontrar novas estratégias e recursos para desenvolver o que lhe foi passado (Camacho et al.,2019).

\subsubsection{Descrição das Etapas do Guia Interativo}

A elaboração do produto educacional seguiu as seguintes etapas: concepção da ideia, escolha do público-alvo, estabelecimento do objetivo, e estabelecimento do orçamento. Em seguida foi definido as etapas e temas a serem abordados, estrutura de textos, bem como definição dos vídeos a serem incluídos e de artigos para fundamentar o tema exposto.

O conteúdo explorado foi baseado na literatura científica relacionado aos cuidados e práticas de saúde bucal utilizada em idosos demenciados.

As imagens e sons usados são livres de direitos autorais. Possuem licença Creative Commons (Atribuição - Não Comercial - Compartilha Igual CC BY-NC-AS), que permitem que outros utilizem, adaptem e criem a partir do trabalho original para fins não comerciais, desde que atribuam aos autores o devido crédito e que licenciem as novas criações sob termos idênticos. Algumas imagens são próprias, $100 \%$ originais, criadas pelo designer gráfico contratado.

\subsubsection{Em que Teoria Baseia a Feitura do Guia Interativo?}

Para a construção do produto foi utilizado o método CTM3, baseado em princípios de teorias comportamentais, a análise transacional (estruturação de personalidade), uso e exploração dos sentidos e programação neurolinguística, através da estruturação de âncoras, descritos por Santos, Teixeira, Warren, \& Rocha (2019), em que os autores colocam que cada indivíduo é único, age e reage de uma forma peculiar a cada evento e que se deve saber quais canais estão disponíveis e como acessá-los (consciente ou inconscientemente).

Santos et al. (2019) descrevem a análise transacional criada em 1965 pelo psiquiatra Eric Berne, que propôs uma estruturação de personalidade composta por três Estados de Ego: Pai, Adulto e Criança. Os Estados de Ego representam a maneira como os indivíduos se comportam, dependendo dos pensamentos e emoções do momento, quando os indivíduos se comunicam agem em função de uma dessas três estruturas, que é ativa neles, cada um com características diferentes e diferentes em cada pessoa. Seria ideal que as pessoas tivessem disponibilidade das três estruturas sempre que fossem solicitadas nos momentos de interação.

O Estado de Ego Pai representa regras, normas, limites, cuidados, atenção e aprendizado adquiridos a partir de figuras parentais e ensina ao indivíduo o certo, o errado e como deve agir e em quem acreditar. Já o Estado de Ego Adulto representa o pensamento lógico e o raciocínio. Não há espaço para emoções, é pura racionalidade, em que a informação é analisada e processada de forma limpa e pensamento racional. Por sua vez, o Estado de Ego Criança é responsável pela alegria, prazer, naturalidade, criatividade e espontaneidade e é também responsável pela fantasia (Santos et al., 2019).

O Método CTM3 propõe-se a inserção das âncoras, que funcionam com o objetivo de reforçar e resgatar a memória original, e evocar o comportamento que se deseja (Santos \& Warren, 2020). Esta foi estabelecida pela imagem de um dente composto por um coração, pois remete ao cuidado e carinho com o idoso com demência, que é encorajado pelo produto e que 
podem funcionar, também, como mensagem subliminar, que é o elemento que não comunica diretamente, tendo a capacidade de vencer a resistência em captar a informação, agindo de forma inconsciente.

A inserção multissensorial implica agregar elementos ao produto educacional que evoquem a visão, a audição, o olfato, o paladar e o tato/sinestésico (Santos \& Warren, 2020).

No Quadro 1 descrevemos os elementos inseridos no produto. O Guia Tutorial Interativo: "Práticas de saúde bucal em idosos com demência”, o estado de Ego Adulto pode ser observado nas recomendações como na página 4, onde há uma citação do que é a definição de demência, baseado na literatura. O estado de Ego Pai pode ser observado na página 7, onde são feitas as recomendações das fases do tratamento odontológico em pacientes demenciados, assim como na fala dos profissionais que participam dos vídeos. O estado de Ego criança pode ser observado no vídeo da página 10, onde se ensina a confecção manual de abridores de boca e bonecas, para higienização da cavidade bucal. No guia tutorial a visão é representada pelo guia em geral, a audição pelos vídeos presentes no produto, o olfato e paladar são representados pelos produtos utilizados na higiene bucal com suas figuras representativas e o tato no vídeo da página 10, onde utilizamos as mãos para confecção de artifícios para auxiliar no atendimento odontológico de pacientes demenciados.

Quadro 1 - Desenho metodológico do Produto Educacional.

\begin{tabular}{|c|c|c|c|}
\hline ETAPA & \multicolumn{2}{|c|}{ DEFINIÇÃO } & DESCRITIVO \\
\hline \multirow{2}{*}{$\mathbf{C}$} & \multicolumn{2}{|l|}{ Concepção } & Guia Tutorial Interativo \\
\hline & \multicolumn{2}{|l|}{ Tema } & Práticas de saúde bucal em idosos com demência \\
\hline $\mathbf{T}$ & \multicolumn{2}{|l|}{ Referencial Teórico } & \\
\hline \multirow{10}{*}{ M3 } & Teoria & Fundamento & Elementos inseridos no recurso educacional \\
\hline & \multirow{3}{*}{$\begin{array}{c}\text { Análise } \\
\text { Transacional }\end{array}$} & Ego pai & $\begin{array}{l}\text { Página } 7 \text { do tutorial, onde são dadas as recomendações das fases do tratamento } \\
\text { das demencias. } \\
\text { Fala dos profissionais que participam do vídeo. }\end{array}$ \\
\hline & & Ego adulto & $\begin{array}{l}\text { Conceito de demência } \\
\text { Recomendações (página 4, do tutorial) } \\
\text { Artigos científicos inseridos }\end{array}$ \\
\hline & & Ego criança & $\begin{array}{l}\text { Pode ser observado na página } 09 \text {, onde ensina-se a confecção dos abridores e } \\
\text { bonecas para higiene bucal; Vídeo animado página } 10 .\end{array}$ \\
\hline & Neurolinguística & $\hat{A} n c o r a$ & Imagens do dente composto por um coração. \\
\hline & \multirow{5}{*}{ Multissensorialidade } & Visão & Guia tutorial em geral \\
\hline & & Audição & Vídeos presente no produto educacional \\
\hline & & Olfato & Representados pelos produtos de higienização bucal, pasta, colutório \\
\hline & & Gustativo & Representados pelos produtos de higienização bucal, pasta, colutório \\
\hline & & Sinestésico & Ação de criar os abridores de boca e bonecas de higiene bucal. \\
\hline
\end{tabular}

Fonte: Autores (2022).

\section{Resultados}

Um produto educacional é um instrumento didático-pedagógico introduzido no contexto da pesquisa, que visa auxiliar o trabalho docente, onde os dados de sua efetivação sejam orientadoras do processo analítico da dissertação do mestrado profissional. Esse produto foi validado na banca de defesa de Mestrado Profissional Ensino em Saúde e Tecnologia, sem sugestões de alteração do seu formato original, na ocasião foi emitido o parecer validado sem alterações, a versão final foi publicada no Portal EduCAPES no endereço eletrônico: http://educapes.capes.gov.br/handle/capes/602716.

O guia é composto por vídeos que versam sobre a definição de demência, a abordagem odontológica a idosos com demência, a importância dos cuidados gerais e de saúde bucal, a importância de higiene bucal, os recursos que auxiliam para realizar a higiene oral de pacientes não colaborativos, a confecção de dispositivos para auxiliar na higiene bucal, os cuidados com as próteses dentárias e a importância do atendimento interdisciplinar. 
Os vídeos foram produzidos por profissionais dentistas e terapêutica ocupacional, discentes e docentes do Mestrado Profissional Ensino em Saúde e Tecnologia da UNCISAL, docente e coordenadora da Especialização em Odontogeriatria da IOA, docente da UNIFACEX, docentes da UNIESP e profissionais que trabalham em áreas afins com o tema do produto.

O primeiro tema abordado no guia, relata o método em que foi estruturado, seguido pela apresentação propriamente dita do guia tutorial, em seguida tem-se um especialista que aborda os diferentes tipos de demências facilitando assim o diagnóstico. Dando continuidade com a fala sobre a abordagem odontológica do idoso com demência, em que se disponibiliza artigo embasando a fala. Prossegue com a importância da higiene bucal em especial a esses idosos, o que se tem disponível para execução dessa higiene, assim como ensina-se a confecção de dispositivos para sua realização. Fala-se, ainda, sobre os cuidados com as próteses e sobre a importância da equipe multidisciplinar no tratamento desses idosos demenciados.

O uso do Guia Interativo Digital, proporciona que o aprendiz seja atuante na concepção do seu saber, argumentando suas percepções, fazendo-se livre, associando situações, intervindo quando necessário, ampliando sua criatividade, sendo proativo e assim formando respostas para adversidades.

\section{Discussão}

Os pacientes idosos com demência devem ter a sua higiene e a sua saúde bucal melhoradas, podendo ser lançado estratégias de educação em saúde com cuidadores formais e informais, uso de ferramentas de triagem de saúde bucal, bem como atendimento odontológico regular (Delwel et al. 2017).

No estudo de Philip et al. (2012) foi identificado como um fator de risco para saúde bucal a assistência de higiene oral e dependência funcional deficiente entre os residentes com demência, além de questionar a capacidade e qualidade dos profissionais em saúde fornecer cuidados orais eficazes onde corroboram com os 31 achados de Araújo, Andrade \& Pinto (2020), no qual identificou que pacientes idosos com alguma síndrome demencial apresentam fragilidades que dificultam e/ou impedem a realização de uma higiene bucal de qualidade.

Ferreira et al. (2014), ao realizar um estudo com o objetivo de identificar a prevalência de idosos com comprometimento cognitivo e identificar associações entre este e as condições de saúde bucal, observou que indivíduos com comprometimento cognitivo apresentaram piores condições de saúde bucal do que os idosos com condição cognitiva preservada, incluindo cárie coronária, cárie radicular, raízes retidas, bolsas periodontais, lesões mucosas. Esses achados confirmam os desfechos do estudo de Philips et al. (2012) e revelou que o estado de higiene oral e gengival era pobre entre os residentes das ILPs com demência, em comparação com aqueles sem demência e que os residentes incapacitados e com demência tinham piores escores em relação ao restante da amostra.

$\mathrm{Na}$ produção de produtos educacionais em saúde, as habilidades e o reconhecimento das necessidades sociais são primordiais, assim como o uso das tecnologias da informação e comunicação, vídeos têm uma importância singular na contribuição para a construção de vínculos entre pacientes e profissionais de saúde, facilitando o processo educacional através de uma comunicação dialógica, com objetivo de troca de saberes para facilitar mudanças de comportamentos, autonomia e melhoria na qualidade de vida (Leandro et al. 2021).

Diante dessas realidades, o crescimento da população idosa e a necessidade de assistência no território corrobora para o investimento público em treinamentos para as equipes de saúde, com ênfase em colaboração multiprofissional, e destacandose que os profissionais da Odontologia podem contribuir para orientações de familiares e cuidadores, com o objetivo de manter a higiene oral e reduzir a perda dentária, assim como presença de dor e outros agravos ocorridos em consequência da ausência do cuidado (Barbosa et al. 2021). 


\section{Considerações Finais}

Esta proposta de produto educacional apresenta instrumentos que buscam facilitar o processo de ensinoaprendizagem, através do estabelecimento de práticas educativas, instruindo cuidadores e dentistas quanto as práticas de saúde bucal em pacientes com demência, utilizando a tecnologia como moderador do processo e estimulando a busca por educação continuada, além de estimular a maior resolutividade de problemas na Atenção Primária de Saúde. Estas tecnologias inseridas no processo de ensino e aprendizagem facilitam a aquisição de informações, pois a criação desse produto possui a capacidade de impactar a qualidade de vida do idoso demenciado.

Espera-se que esse manual impacte sobre a vida de idosos demenciados, de forma a contribuir na instalação de bons hábitos, assim como no treinamento de cuidadores, familiares. E, por último, não menos importante sugere-se novas pesquisas na área utilizando a tecnologia como instrumento facilitador de processos educacionais, sendo produzidos em variados cenários, com diversos temas, bem como em diferentes níveis de atenção à saúde

\section{Referências}

Araujo, A. S., Andrade, M., \& Pinto, F. M. A. G. (2020). Higiene e saúde bucal em idosos na atenção primária: uma revisão sistemática. Revista Eletrônica Acervo Saúde, (44), e2673. https://doi.org/10.25248/reas.e2673.2020

Brasil. (2006). Cadernos de Atenção Básica, n. 19 - Envelhecimento e saúde da pessoa idosa. Ministério da Saúde.

Brasil. (2007). Diretrizes de educação em saúde visando à promoção da saúde: documento base. Funasa, (Documento I).

Barbosa, E. P., Meneses P. V. S., Novaes, C. R. M. N., Leandro, A. R. L., Assis, V. L. B., \& Reis, M. C. S. (2021). Práticas de saúde oral em idosos com demência: Revisão sistemática. Research, Society and Development, 10(9), e59510918367. https://doi.org/10.33448/rsd-v10i9.18367

Boulet, A. (2011). Le manuel scolaire numérique, produit éditorial et outil documentaire à valeur ajoutée: anatomie d'un concept en développement, enjeux et perspectives de son intégration dans les pratiques éducatives. LE CNAM; INTD.

Brunetti, R. F., \& Montenegro, F. L. B. (2002). Odontogeriatria: noções de interesse clínico. Artes Médicas.

Camacho, A. C. L. F., Capetini, A. C., Guimarães, A. O., Santos, A. C. F. T., Silva, A., P., \& Andrade, G. N. (2019). Tecnologia educacional interativa sobre cuidados a idosos com demências. Revista de Enfermagem UFPE, 13(1), 249- 254.

Cancela, D. M. G. (2007). O processo de envelhecimento. Psicologia.com.pt: o Portal dos Psicólogos. Recuperado em 10 de agosto, 2021, de: http://www.psicologia.pt/artigos/textos/TL0097.pdf

Delwel, S., Binnekade, T. T., Perez, R. S., Hertogh, C. M., Scherder, E. J., \& Lobbezoo, F. (2017). Oral health and orofacial pain in older people with dementia: a systematic review with focus on dental hard tissues. Clinical oral investigations, 21(1), 17-32. https://doi.org/10.1007/s00784-016-1934-9

Fereshtehnejad, S. M., Garcia-Ptacek, S., Religa, D., Holmer, J., Buhlin, K., Eriksdotter, M., \& Sandborgh-Englund, G. (2018). Dental care utilization in patients with different types of dementia: A longitudinal nationwide study of 58,037 individuals. Alzheimer's \& dementia: the journal of the Alzheimer's Association, 14(1), 10-19. https://doi.org/10.1016/j.jalz.2017.05.004

Ferreira, R. C., Vargas, A. M. D., Fernandes, N. C. N., Souza, J. G. S., Sá, M. A. B., Oliveira, L. F. B., \& Martins, A. M. E. B. L. (2014). O idoso com comprometimento cognitivo apresenta pior condição de saúde bucal? Ciências \& Saúde Coletiva, 19(8), 3417-3428. Recuperado em 10 de agosto, 2021, de: https://www.scielo.br/j/csc/a/6XyVLpdfWH3ShG6gMgPHGmg/?format=pdf\&lang=pt

Franco, J. F., Cruz, S. R. R., \& Lopes, R. D. (2007). Experiências de Uso de Mídias Interativas como Suporte para Autoria e Construção Colaborativa do Conhecimento. RENOTE, 5(1), 1-11. https://doi.org/10.22456/1679-1916.14136

Grossberg, G. T. (2003). Diagnosis and Treatment of Alzheimer's disease. The Journal of clinical psychiatry, 64(9), 3-6.

Laginestra-Silva, A., Tuyama, F. L. G., Cerceau, V. R., Mariano, T. D. C. A.., Pinheiro, H. A., \& Oliveira, M. L. C. de. (2021). Prevalência de demências no Brasil: um estudo de revisão sistemática. Revista Neurociências, 29, 1-14.

Leandro, A. R. L., Novaes, C. R. M. N., Barbosa, E. P., Meneses, P. V. de S., Sales, M. R. B., \& Bandini, H. H. M. (2021). Construction of educational products on the rational use of medicines. Research, Society and Development, 10(14), e495101422232. https://doi.org/10.33448/rsd-v10i14.22232

Organização Mundial da Saúde (2019). Redução do risco de declínio cognitivo e demência: diretrizes da OMS. Genebra: OMS.

Miranda, A. F., Costa, C. R., Franco, E. J., Alves, V. P., \& Montenegro, F. L. B. (2018). Diretrizes e desafios num atendimento odontológico de pacientes com Alzheimer em estágio avançado. Revista de Medicina e Saúde de Brasília, 7(2), 285-295.

Newton, J. P. (2007). Dementia, oral health and the failing dentition. Gerodontology, 24(2), 65-66.

Paim, J., Travassos, C., Almeida, C., Bahia, L., \& Macinko, J. (2011). Sistema de saúde brasileiro: história, avanços e desafios. Lancet, 3, 11-31. 
Research, Society and Development, v. 11, n. 3, e39511326658, 2022

(CC BY 4.0) | ISSN 2525-3409 | DOI: http://dx.doi.org/10.33448/rsd-v11i3.26658

Philip, P., Rogers, C., Kruger, E., \& Tennant, M. (2012). Oral hygiene care status of elderly with dementia and in residential aged care facilities. Gerodontology, 29(2), e306-e311. https://doi.org/10.1111/j.1741-2358.2011.00472.x

Santiago, E., Simões, R. J. P., \& Pereira, J. A. L. (2008) A Saúde Oral na Doença de Alzheimer. Arquivo de Medicina, 22(6), 189-193.

Santos, A. A.; \& Warren, E. M. C. (2020) Método CTM3 como Dispositivo de Ensino, Aprendizagem e Comunicação em Produtos Educacionais. In: Santos, A. A. dos (org.). Educação em saúde: trabalhando com produtos educacionais. (pp. 12-29). Maceió: Hawking.

Santos, A. A., Teixeira, G. M., Warren, E. M. C., \& Rocha, M. F. M. R. (2019). Saúde na Infância Bucal e a Contribuição dos Recursos Educacionais. In Oliveira, W. A., Torales, A. P. B., Barros, L. M., Iturribarria, G. M., \& Oliveira, C. C. C. (Org.). Perspectivas em saúde coletiva: modelos e práticas interdisciplinares. (pp. 275-285). Curitiba: CRV

Sousa, R. P., Miota, F. M. C. S. C., \& Carvalho, A. B. G. (org.). (2011). Tecnologias digitais na educação. Campina Grande: EDUEPB.

Zenthofer, A., Schrõder, J., Cabrera, T., Rammelsberg, P., \& Hassel, A. J. (2014). Comparison of oral heath among older people with and without dementia. Community Dental Health Journal, 31(1), 27-31. 